Barcelona, Spain, November 17 - 20, 2013

\title{
Research on Simplification of Simulating the Heat Conduction in the Lithium-ion Battery Core
}

\author{
Xuning FENG ${ }^{1}$, Xiangming $\mathrm{HE}^{1,2}$, Languang $\mathrm{LU}^{1}$, Minggao OUYANG ${ }^{1, *}$ \\ 1. State Key Laboratory of Automotive Safety and Energy, Tsinghua University, Beijing 100084, China. \\ *Corresponding Author,ouymg@tsinghua.edu.cn \\ 2. Institute of Nuclear and New Energy Technology, Tsinghua University, Beijing, 100084, China
}

\begin{abstract}
This paper discusses the model simplifing issue in battery thermal simulation. The paper verifies that for the large power battery simplifying the multilayer battery core as a lumped cuboid is reasonable. So when doing simulation, building a multilayer core is unnecessary. And the calculation cost can be reduced by the lumped model. Specific power battetry of $70 \mathrm{Ah}$ is dissembled to be modeld. Thermal models of are established, including models with a lumped core and with multilayer cores. For the lumped core, the anisotropic thermal conductivities are got by equations calculating series and parallel equivalent thermal conductivity. While for the multilayer core models, the core contains numbers of unit cells and the volume of which is equal to that of the lumped. In addition, under the boundary conditions of inner heat source and surface heating, steady state simulations are performed. Simulation results indicate that the temperature distributions of the lumped model and the multilayer model are almost the same. For one thing, large number of multilayers and low shell thermal conductivity contribute to a uniform temperature distribution within the core, so it is reasonable to simplify the multilayer core as a lumped cuboid. For another, due to the size of the battery and the shell property, it is difficult to find a simple curve to fit the simulation temperature on the battery surface. Although minor differences still exist, the lumped core can well subsitute the multilayer core in battery thermal simulation.
\end{abstract}

\section{Key Words}

Lithium ion battery; thermal modeling; multilayer; core simplification; anisotropic conductivity 


\section{Introduction}

Under the pressure of energy crisis and environmental protection, electric vehicle is attracting wide spread interest. Lithium-ion battery is a promising power source for the electric vehicle. Since thermal condition greatly influences the performance of the lithium-ion battery, there is a need for a good understanding of lithium-ion battery thermal issues [1]. Thermal modeling is a prevailing method to do research on battery thermal issues. The battery consists of the core, the shell, the terminal, the safety vent etc $[2,3]$. Core modeling is the crucial part of thermal modeling. There are two common types of battery core: the prismatic type [2-6] and the cylinder type [7-13]. The battery has a layered core. To guarantee the capacity of the battery, each battery consists of some unit cells. Generally speaking, each unit cell contains two electrodes, two collectors and a separator. In some modeling cases, the core is modeled as a multilayer structure [6-9], while in some others, the core is modeled as a lumped cuboid with anisotropic thermal conductivity $[2-5,11]$. Simulation model of multilayer structure requires larger amount of calculation than that of lumped cuboid. In many cases when building a model of lumped cuboid, the equivalent thermal conductivity in perpendicular and parallel directions are calculated by classic heat transfer equations of series and parallel equivalent thermal conductivity for composite slabs[2-4, 10]. However, in the text books of heat transfer, the equations for calculating equivalent thermal conductivity works only when the thermal conductivities of different layers have little difference[14, 15]. While in battery core modeling, the thermal conductivity of different materials differ on several times. Therefore there is a need to verify the reasonableness of the simplifying method by the equations of series and parallel thermal conductivity for composite slabs.

In this paper, 3D numerical models of battery core with different number of unit cells are established to do simulations. Three different thermal boundary conditions are set to perform the simulation. Simulation results are analyzed to come to a conclusion that it is reasonable to simplify the multilayer core into a lumped cuboid with anisotropic thermal conductivity.

\section{Model assumption}

\subsection{Parameters}

A 70Ah $\mathrm{LiFePO}_{4}$ battery(Fig. 1a) produced by CALB(China Aviation Lithium Battery) is dissembled to get the parameters of the core. 


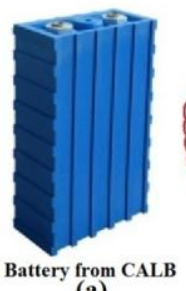

(a)

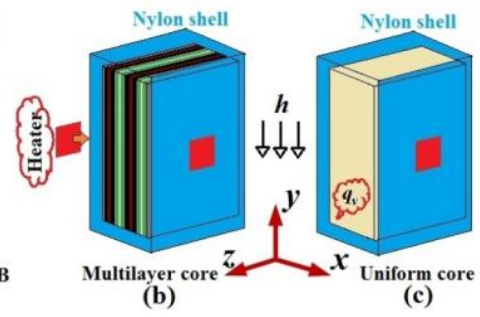

(c)
Figure 1: 70Ah $\mathrm{LiFePO}_{4}$ battery used for modeling

Before dissembled, the battery is discharged to 0

SOC(State of Charge) to ensure safety. The geometry parameters of the core is measured by a micrometer (Table 1). The measured size of the core is $50.192 * 100 * 170 \mathrm{~mm}$. To simplify the model, the size of the core is normalized to 50*100*170 mm. The "Normalization" in Table 1 means the thicknesses of different materials are all normalized to meet total thickness of $50 \mathrm{~mm}$. To eliminate the influence of the terminals and make reasonable boundary conditions of the battery core, the model only has a core surrounded by a nylon shell with a thickness of 5mm(Fig. 1b, Fig. 1c). The physical parameters collected from references $[1,16,17]$ are listed in Table 2. By employing equation(1) and (2), the anisotropic thermal conductivity both in series and in parallel direction can be calculated.

$$
\begin{gathered}
\lambda_{x}=\frac{L_{x}}{\sum_{i} L_{x_{i}} / \lambda_{i}}=0.983 \mathrm{~W} \cdot \mathrm{m}^{-1} \cdot \mathrm{K}^{-1} \\
\lambda_{y}=\lambda_{z}=\left(\sum_{i} \lambda_{i} L_{x_{i}}\right) / L_{x}=38.54 \mathrm{~W} \cdot \mathrm{m}^{-1} \cdot \mathrm{K}^{-1}
\end{gathered}
$$

In addition, calculating the specific density and the specific heat capacity of the lumped core:

$$
\begin{gathered}
\rho_{\text {Total }}=\left(\sum_{i} \rho_{i} L_{i}\right) / L_{\text {Total }}=2197 \mathrm{~kg} \cdot \mathrm{m}^{-3} \\
c_{p, \text { Total }}=\left(\sum_{i} \rho_{i} c_{p i} L_{i}\right) / \rho_{\text {Total }} L_{\text {Total }}=1193 \mathrm{~J} \cdot \mathrm{kg}^{-1} \cdot \mathrm{K}^{-1}(4)
\end{gathered}
$$

Both the multilayer and lumped core models are founded in GAMBIT $^{\circledR}$. The multilayer model contains numbers of unit cells. A unit cell contains layers of graphite, copper foil, separator,

Table 1 Measured specifications of the battery core

\begin{tabular}{ccccccc}
\hline & Graphite & Copper foil & Separator & $\mathrm{LiFePO}_{4}$ & Aluminum foil & Total \\
\hline Single-layer thickness/ $\mu \mathrm{m}$ & 72 & 20 & 40 & 90 & 40 & - \\
Number of layers & 216 & 108 & 218 & 216 & 108 & - \\
All-layer thickness/mm & 15.552 & 2.16 & 8.72 & 19.44 & 4.32 & 50.192 \\
After normalization/mm & 15.49 & 2.15 & 8.69 & 19.37 & 4.3 & 50 \\
\hline
\end{tabular}

Table 2 Physical parameters collected from references [1][16][17]

\begin{tabular}{ccccccc}
\hline & $\begin{array}{c}\text { Graphit } \\
\mathrm{e}\end{array}$ & $\begin{array}{c}\text { Copper } \\
\text { foil }\end{array}$ & $\begin{array}{c}\text { Separato } \\
\mathrm{r}\end{array}$ & $\begin{array}{c}\text { LiFePO } \\
4\end{array}$ & $\begin{array}{c}\text { Aluminum } \\
\text { foil }\end{array}$ & $\begin{array}{c}\text { Nylon } \\
\text { shell }\end{array}$ \\
\hline Total & 15.49 & 2.15 & 8.69 & 19.37 & 4.3 & 5 \\
thickness/mm & 2660 & 8933 & 1008 & 1500 & 2702 & 1180 \\
$\rho\left(\mathrm{kg} \cdot \mathrm{m}^{-3}\right)$ & 1437 & 385 & 1978 & 1260 & 903 & 1500 \\
$c_{p}\left(\mathrm{~J} \cdot \mathrm{kg}^{-1} \cdot \mathrm{K}^{-1}\right)$ & 1.04 & 398 & 0.38 & 1.48 & 238 & 0.35 \\
$\lambda\left(\mathrm{W} \cdot \mathrm{m}^{-1} \cdot \mathrm{K}^{-1}\right)$ & 1.48 & \\
\hline
\end{tabular}




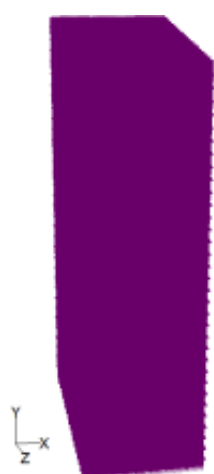

(a)

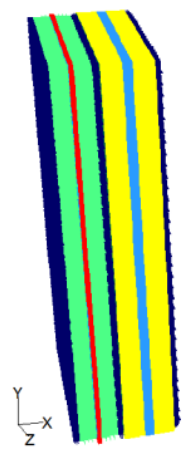

(b)

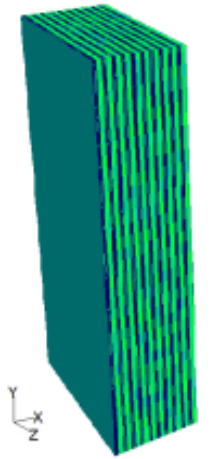

(c)
Figure 2: Core model with different number of unit cells, (a) 0 unit cell, (b) 1 unit cell, (c) 10 unit cells.

The number of unit cell for the lumped core is 0 .

While the numbers employed in multilayer modeling are 1, 2, 10 and 20 (Fig. 2). It should be noted that we can not apply more multilayer numbers to do such a simulation, because the thickness of each layer will be too small to be meshed for simulation. In the following part, it can be seen that the temperature distribution for the 20-layer core is quite uniform without fluctuation, and the simulation results for 10-layer core and 20-layer core is quite similar, so simulations for more multilayer number is meaningless.

\subsection{Boundary conditions}

The control function can be described as (5)

$$
0=\lambda_{x} \frac{\partial^{2} T}{\partial x^{2}}+\lambda_{y} \frac{\partial^{2} T}{\partial y^{2}}+\lambda_{z} \frac{\partial^{2} T}{\partial z^{2}}+q_{v}
$$

Steady state simulations are done in FLUENT under three kinds of boundary conditions(Fig. 1b\&c): (1) uniform inner heat source all over the core; (2) or (3) isothermal heat source on the shell surface in positive or negative $x$ direction, like a heater pasted on the outer surface of the shell. The shell works under an assuming convection condition with an average coefficient of $h=4 \mathrm{~W} \cdot \mathrm{m}^{-2} \cdot \mathrm{K}^{-1}$. The ambient temperature is $293 \mathrm{~K}$. The reason for setting the first boundry condition is that the $1 \mathrm{C}$ test in laboratory is most common. The reason for setting the second and third boundry condition is that we have developed a method to get the thermal conductivity by pasting a heater onto the surface of the battery [18]. However, this battery is thicker than that in [18], we wish to study whether it is reasonable to apply that method again.

Assuming the average internal resistance of the battery is $1.5 \mathrm{~m} \Omega$ by our experience on measuring the specific battery [3]. Since the 1C current of the battery is $70 \mathrm{~A}$, the $1 \mathrm{C}$ Joule heating source should be $Q=I^{2} R=70^{2} \times 0.0015=7.35 \mathrm{~W}$. And the inner heat source $\quad q_{v}=Q / V=7.35 /(0.17 \times 0.1 \times 0.05)=8647$ $\mathrm{W} / \mathrm{m}^{3}$. In addition, when doing simulation under surface heating conditions (2) and (3), the isothermal temperature of the heater is $323 \mathrm{~K}$. Because the heat flow under condition (2) or (3) may spread from graphite to $\mathrm{LiFePO}_{4}$, or from 
Table 315 groups of simulation results

\begin{tabular}{|c|c|c|c|}
\hline $\mathrm{P}_{\text {Bonditions }}^{\text {No. of }}{ }^{\text {unit }}$ & 0 & 1 & 2 \\
\hline Inner heat source & & & \\
\hline Heating from $x^{+}$direction & & & \\
\hline Heating from $x^{-}$direction & & & \\
\hline
\end{tabular}

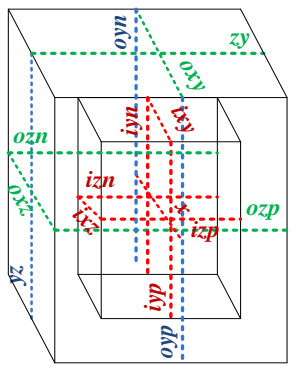

(a)

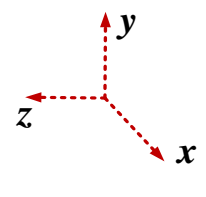

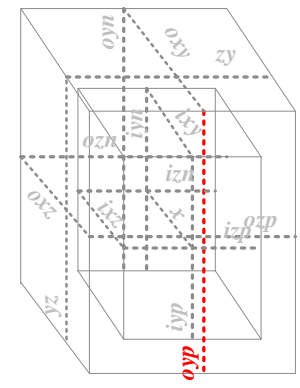

(b)

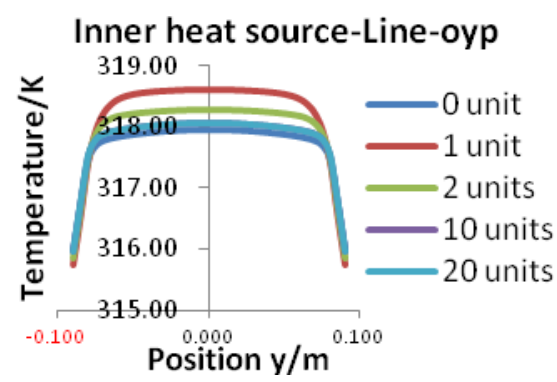

(c)

Figure 3 Schematic maps of characteristic lines

$\mathrm{LiFePO}_{4}$ to graphite, the heater is set on the positive and negative $x$ direction respectively to find the difference.

\section{Data Processing}

As mentioned in Section 2, 5 cores with different numbers of unit cells and 3 boundary conditions lead to 15 groups of simulation results(Table 3). Each group contains 3D data of temperature distribution. To do quantitative comparisons among different groups of results, 15 characteristic lines are defined(Fig. 3a). To be specific, in the naming of characteristic lines, $i$ means the core surface, $o$ means the shell surface, $p$ represents $x$ positive direction and $n$ represents $x$ negative direction. And $x$ represents that the characteristic line locates on a surface with norm vector $x$. For example, line-oyp means that the line locates at the outer surface of the shell at the $x$ positive direction. And $y$ axis is the norm vector of the surface(Fig. 3b). What's more, the temperature distribution on line-oyp of different numbers of unit cells are plotted in Fig. 3c.

\section{Results and discussions}

In Table 3, it can be seen that the average 


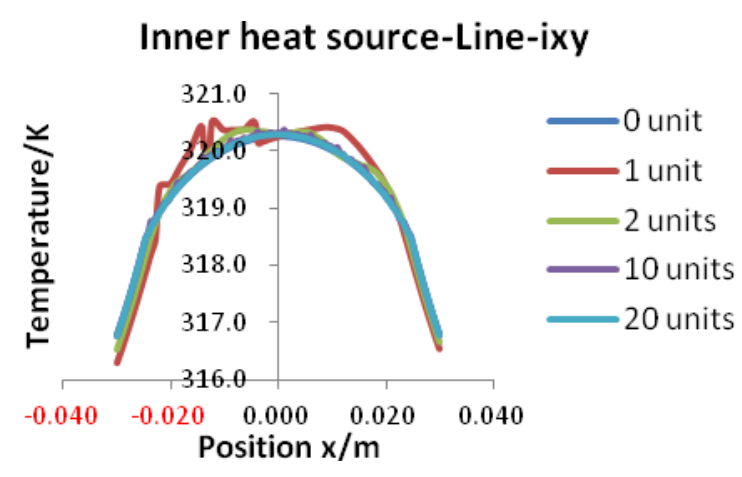

(a)

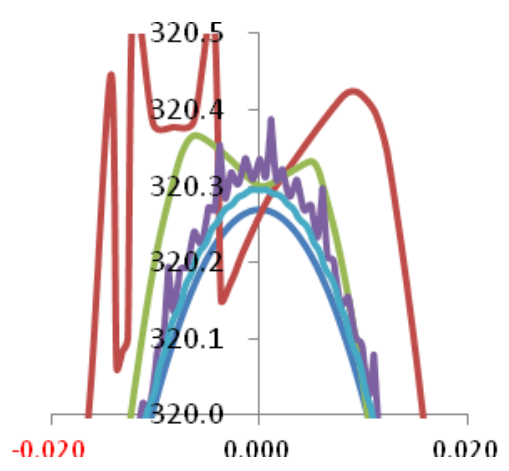

(b) Magnified map of(a)

Figure 4 Relationship between number of unit cells and the temperature fluctuations

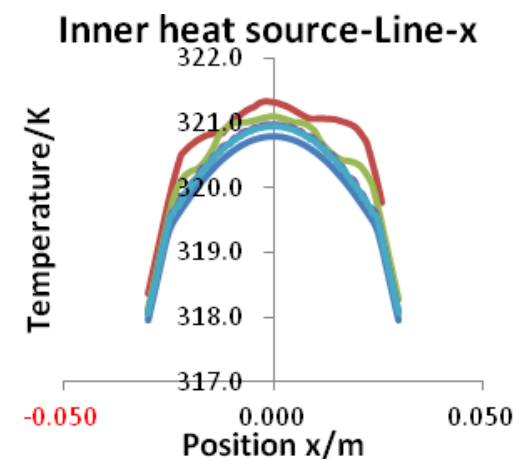

(a)

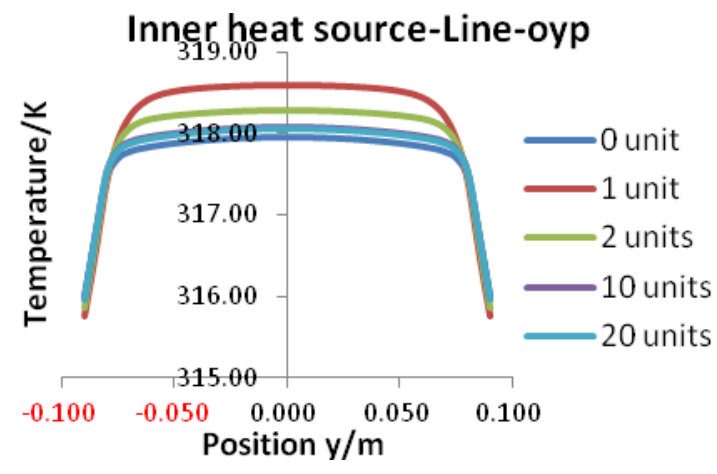

(b)

Figure 5 Relationship between the number of unit cells and the peak temperature

temperature on the surface perpendicular to $y \& z$ axis is about $1^{\circ} \mathrm{C}$ higher than that of $x$ axis, when the battery is inner heated. Moreover, when heated from shell surface, high temperature intensed districts occur on the surface with norm $y \& z$ in models with the lumped core and core of 10 or more unit cells.

\subsection{Results under inner heat source}

(1) Relationship between number of unit cells and the temperature fluctuations

In simulation, when the number of unit cells is small, the temperature fluctuations are obvious. However, fluctuations recede when the number of unit cell increase(Fig. 4).

(2) Relationship between the number of unit cells and the peak temperature

Despite the temperature fluctuations, when the $x$ coordinate is zero, the temperature reaches a maximum value. But the maximum value decreases as the number of unit cells increases. What's more, the decreasing rate also decreases(Fig. 5). 


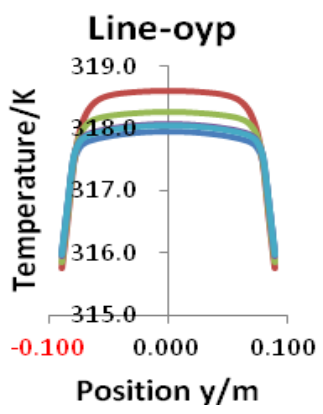

(a)

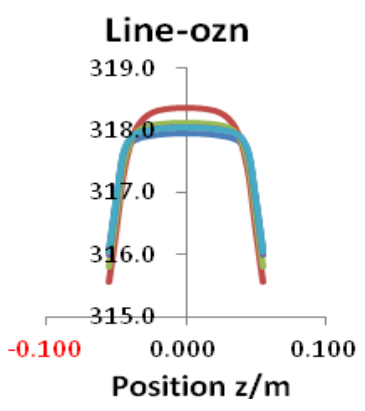

(b)

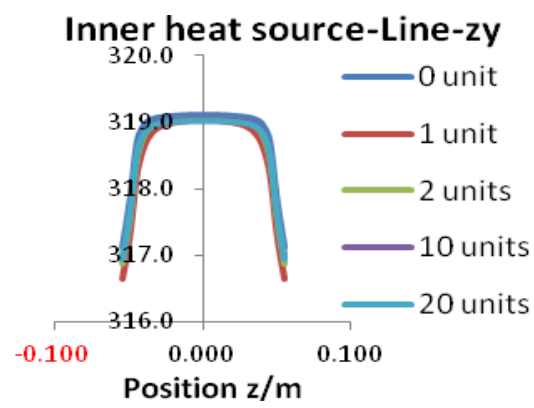

(c)

Figure 6 Difference between the lumped core and the multilayer cores when inner heated
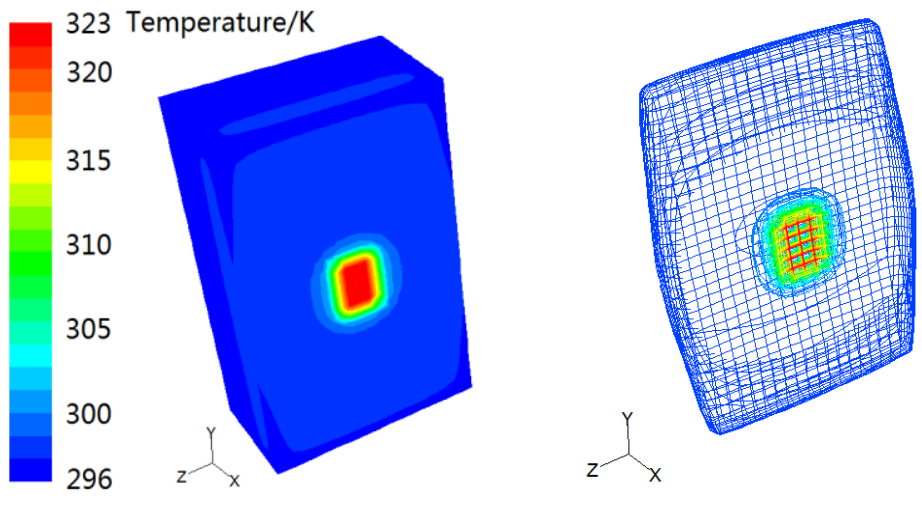

Figure 7 Spread of heat flow in $y \& z$ direction

\section{(3) Difference between the lumped core and}

\section{the multilayer cores}

In spite of the temperature fluctuations in multilayer cores, the average temperature on the surface with norm $x$ of the lumped model is less than that of the multilayer cores (Fig. 6). That result means that the thermal conductivity in $x$ direction of the lumped core is a little less than that of the multilayer core. In other words, the thermal conductivity in $y \& z$ direction of the lumped core is relatively bigger than that of the multilayer core.

\subsection{Results under surface heating}

(1) Difference between heating from positive

\section{or negative $x$ direction}

When the heat source is set at the $x$ positive direction on the shell surface, the heat flow will pass from the $\mathrm{LiFePO}_{4}$ layer to the graphite. While for heater at the $x$ negative direction, the heat flow will start at the graphite layer, the thermal conductivity of which is higher than that of the $\mathrm{LiFePO}_{4}$ layer. However, the temperature difference is very small alone line $x$, with a $0.3^{\circ} \mathrm{C}$ average temperature difference.

\section{(2) Spread of the heat flow in $y \& z$ direction}

Although the heat source is set at the terminal surface alone the $x$ direction, the heat flow also spreads in $y \& z$ direction (Fig. 7). In 

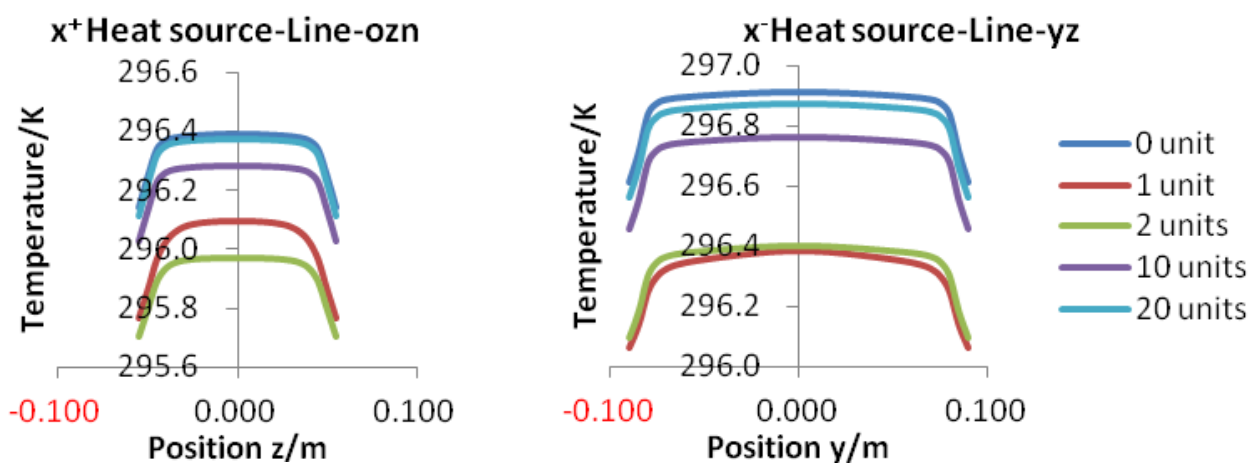

Figure 8 Difference between the lumped core and the multilayer cores under surface heating

supplement, the heat flow spreads much faster in $y \& z$ direction than that in $x$ direction. In addition, temperature peak values occur at the surface with norm $y \& z$, respectively.

(3) Difference between the lumped core and the multilayer cores

Under the boundary conditions of the heat source, the average temperature of the lumped core model is a little bigger in all directions than that of the multilayer core (Fig. 8). In other words, in all directions, the thermal conductivity of all directions of the lumped core may be a little bigger than that of the multilayer cores. In comparison with the conclusion of 4.1(3), $\lambda_{x}, \lambda_{y}$ and $\lambda_{z}$ of the lumped core are all a little larger than that of the multilayer cores, while the thermal conductivity in $y \& z$ direction is much bigger, i.e. $\left(\lambda_{0} / \lambda_{20}\right)_{y, z}>\left(\lambda_{0} / \lambda_{20}\right)_{x}>1$. The subscript 0 and 20 represents the number of unit cells.

\subsection{Discussions}

(1)Trials of curve fitting under surface heating conditions

Once a simple curve can fit the surface temperature distribution of the battery, the battery properties can be well interpreted by surface heating(in situ detection or nondestructive detection) with curve fitting. In other words, curve fitting is worthy for acquiring the thermal properties of lithium-ion batteries. However, in this case, two main factors hinders the application of the curve fitting. First, the value of length, broad and height is close(18:11:5.5). Second, the shell with low thermal conductivity obstructs the spread of heat flow in $y \& z$ direction.Both of those factors make the temperature distribution on the battery surface deform from functions or solutions in classic heat transfer. In fact, the temperature curve cannot be fitted under a common method provided in MATLAB curve fitting toolbox with 


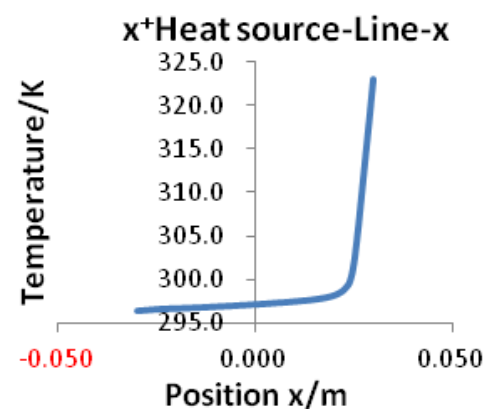

(a)

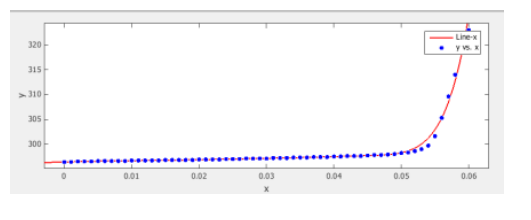

(b)Fitted by polynomial, order $=9$

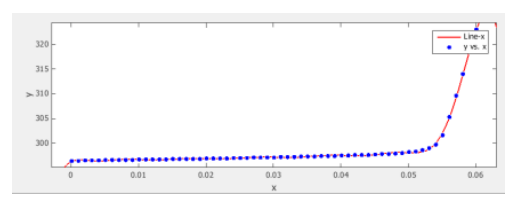

(c)Fitted by sum of sine, order=7

Figure 9 Trials of curve fitting under surface heating conditions

Table 4 Comparison of temperature difference before and after adjustment

\begin{tabular}{c|cccccc}
\hline $\begin{array}{c}\text { Boundry } \\
\text { condition }\end{array}$ & $\begin{array}{c}\text { Characteristic } \\
\text { line }\end{array}$ & $\begin{array}{c}\text { Maximum } \\
\text { temperature of } \\
\text { 20-layer core }(\mathrm{K})\end{array}$ & $\begin{array}{c}\text { Lumped } \\
\text { core initial } \\
(\mathrm{K})\end{array}$ & $\begin{array}{c}\text { Difference } \\
\text { before } \\
\text { adjustment }(\mathrm{K})\end{array}$ & $\begin{array}{c}\text { After } \\
\text { adjustment } \\
(\mathrm{K})\end{array}$ & $\begin{array}{c}\text { Difference } \\
\text { after } \\
\text { adjustment (K) }\end{array}$ \\
\hline $\begin{array}{c}\text { Inner } \\
\text { heating }\end{array}$ & oyp & 318.06 & 317.95 & -0.11 & 318.07 & 0.01 \\
source & yz & 319.08 & 319.18 & 0.10 & 319.06 & -0.02 \\
$x^{+}$heat & oyn & 296.38 & 296.39 & 0.01 & 296.46 & 0.08 \\
source & yz & 296.90 & 296.91 & 0.01 & 296.92 & 0.02 \\
\hline
\end{tabular}

an order less than 7(Fig. 9).

\section{(2)Model adjustments}

In section 4.1(3) and 4.2(3), it is

mentioned that $\left(\lambda_{0} / \lambda_{20}\right)_{y, z}>\left(\lambda_{0} / \lambda_{20}\right)_{x}>1$.

Referring to the simulation results of the core

with 20 unit cells, simulation results by adjusting the thermal conductivity of the lumped core can not well fit that of 20-unit-cell core. However, to break the formula that $\left(\lambda_{0} / \lambda_{20}\right)_{y, z}>\left(\lambda_{0} / \lambda_{20}\right)_{x}$, $\left(\lambda_{0}\right)_{y, z}$ should be decreased and $\left(\lambda_{0}\right)_{x}$ should be increased, so that $\left(\lambda_{0} / \lambda_{20}\right)_{x}$ will be much larger than 1 . That is a contradiction. If the thermal conductivity is changed to $\lambda_{x}^{\prime}=1.15$ $\mathrm{W} \cdot \mathrm{m}^{-1} \cdot \mathrm{K}^{-1}$ and $\lambda_{y, z}^{\prime}=25 \mathrm{~W} \cdot \mathrm{m}^{-1} \cdot \mathrm{K}^{-1}$, the temperature differences in all directions are listed in Table 4. In conclusion, the difference between the lumped core and the multilayer core cannot be eliminated by adjusting thermal conductivity.

What's more, the temperature difference between the lumped core and the multilayer core becomes larger as the magnitude of the uniform heat source goes bigger. However, the maximum temperature difference is relatively low (Table 5).

Table 5 Maximum temperature difference between the lumped core and the multilayer core on the specific characteristic line

\begin{tabular}{l|llll}
\hline Heat source & & & \\
\hline Oyp $\left(\mathrm{W} \cdot \mathrm{m}^{-3}\right)$ & 5000 & 8647 & 15000 & 30000 \\
\hline $\mathrm{Yz}$ & & & & \\
\hline
\end{tabular}




\section{Conclusion}

This paper discusses the model simplifing issue in battery thermal simulation. The paper verifies that simplifying the multilayer battery core as a lumped cuboid is reasonable. So when doing simulation, building a multilayer core is unnecessary. And the calculation cost can be reduced by the lumped model.

3 conclusions are drawn here:

First, large number of multilayer and low shell thermal conductivity contribute to the uniform distribution of core temperature, so it is reasonable to simplify a multilayer core into a lumped cuboid. Second, it is difficult to find an existing function to fit the simulation results on some special points due to the size of the battery and the shell properties. Third, minor differences still exist between the lumped and the multilayer core: $\left(\lambda_{0} / \lambda_{20}\right)_{y, z}>\left(\lambda_{0} / \lambda_{20}\right)_{x}>1$, and the difference cannot be eliminated by adjusting the thermal conductivity of the lumped core.

\section{Acknowledgment}

This work is supported the MOST (Grant No. 2010DFA72760 and Grant No. 2011CB935902), the Tsinghua University Initiative Scientific Research Program (Grand No. 2010THZ08116, No.2011THZ08139 and No.2011THZ01004) and State Key Laboratory of
Automotive Safety and Energy (grand No. ZZ2012-011).

\section{References}

[1] T. M. Bandhauer, S. Garimella and T. F. Fuller, $A$ critical review of thermal issues in lithium-ion batteries, Journal of the Electrochemical Society, ISSN 0013-4651, 158(2011), 1-25.

[2] C. Lin, G. Tian, B. Qiu, Q. Chen, Analysis of influence factors on heat dissipation capability for traction Ni-MH battery, Chinese Journal of Power Sources, ISSN 1002-087X, 32(2008), 115-119(in Chinese).

[3] J. Liang, Research on the heat dissipation of pure EV's battery pack, M.D. Thesis, Tsinghua University, Beijing, Nov. 2011, 18-19.

[4] S.C. Chen, C.C. Wan, Y.Y. Wang, Thermal analysis of lithium-ion batteries, Journal of Power Sources, ISSN 0378-7753, 140(2005), $111-124$.

[5] M. S. Wu, Y.H. Hung, Y.Y. Wang and C.C. Wan, Heat dissipation behavior of the nickel/metal hydrid battery, Journal of the Electrochemical Society, ISSN 0013-4651, 147(2000), 930-935.

[6] Y. Chen, J.W. Evans, Heat transfer phenomena in lithium/polymer-electrolyte batteries for electric vehicle application, Journal of the Electrochemical Society, ISSN 0013-4651, 140(1993), 1833-1838. 
[7] A.A. Pesaran, Battery thermal models for hybrid vehicle simulations, Journal of Power Sources, ISSN 0378-7753, 110(2002), 377-382.

[8] D.H. Jeon, S.M. Baek, Thermal modeling of cylindrical lithium ion battery during discharge cycle, Energy Conversion and Management, ISSN 0196-8904, 52(2011), 2973-2981.

[9] K. Somasundaram, E. Birgersson, A.S. Mujumdar, Thermal-electrochemical model for passive thermal management of a spiral-wound lithium-ion battery. Journal of Power Sources, ISSN 0378-7753, 203(2012), 84-96.

[10] S.C. Chen, Y.Y. Wang, C.C. Wan, Thermal analysis of spirally wound lithium batteries, Journal of the Electrochemical Society, ISSN 0013-4651, 153(2006), 637-648.

[11] A.A. Pesaran, S. Burch, M. Keyser, An approach for designing thermal management systems for electric and hybrid vehicle battery packs, Fourth Vehicle Thermal Management Systems Conference and Exhibition, London, UK, May 24 27, 1999.

[12] S. A. Hallaj, H. Maleki, J.S. Hong and J.R. Selman. Thermal modeling and design considerations of lithium-ion batteries. Journal of Power Sources, ISSN 0378-7753， 83(1999), $1-8$.

[13] C. Forgez, D. V. Do, G. Friedrich et al. Thermal modeling of a cylindrical $\mathrm{LiFePO}_{4} /$ graphite lithium-ion battery, Journal of Power Sources, ISSN 0378-7753, 195(2010), 2961-2968.

[14] X. Zhang, Z. Ren, F. Mei, Heat transfer( $5^{\text {th }}$ ed.), ISBN 7112091836, Beijing, China Architecture \& Building Press, 2007.7, 32.

[15] J.P. Holman, Heat Transfer, ISBN 7111171500 , Beijing, China Machine Press, 2005.7, 26.

[16] G. Guo, B. Long, B. Cheng et al. Three-dimensional thermal finite element modeling of lithium-ion battery in thermal abuse application, Journal of Power Sources, ISSN 0378-7753, 195(2010), 2393-2398.

[17] D.R. Lide. CRC handbook of chemistry and physics, $89^{\text {th }}$ edition, ISBN 1420066791, Boca Raton, CRC Press, 2008-2009.

[18] X. Feng, J. Li, L. Wang et al. Experiments and modeling of anisotropic thermal conductivity of lithium-ion batteries. Journal of Automotive Safety and Energy, ISSN 1674-8484, 3(2012), $158-164$. 


\section{Authors}

Xuning Feng,

\section{Education:}

2011 BS. Department of Automotive

Engineering, Tsinghua University.

\section{Current Activities:}

Battery safety research

Xiangming He,

\section{Education:}

$2007 \mathrm{Ph} . \mathrm{D}$, Insitute of Nuclear and New Energy Technology, Tsinghua University.

\section{Current Activities:}

Advanced materials for lithium ion battery.

Languang $\mathrm{Lu}$,

\section{Education:}

2001 Ph.D, Wuhan University of

Technology.

\section{Current Activities:}

\section{Battery management system}

Online state evaluation of lithium ion

\section{Minggao Ouyang}

\section{Education:}

1993 Ph.D, Technical University of

Denmark.

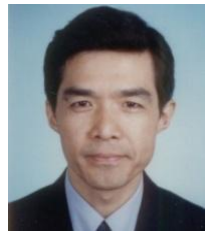

\section{Current Activities:}

Dynamics, models and control of renewable energy powertrain.

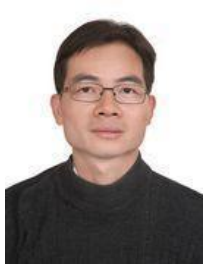

battery 